\title{
Alterações hematológicas em pacientes com dengue
}

\author{
Hematological abnormalities in patients with dengue
}

\author{
Éveny Cristine Luna de Oliveira ${ }^{1}$, Elenir Rose Jardim Cury Pontes ${ }^{2}$, \\ Rivaldo Venâncio da Cunha ${ }^{3}$, Íris Bucker Fróes ${ }^{1}$ e Delso do Nascimento ${ }^{1}$
}

\begin{abstract}
RESUMO
Dengue é uma doença negligenciada de alta morbidade e mortalidade em crianças e adultos, ocorrendo principalmente em regiões tropicais e subtropicais. 0 objetivo desse trabalho foi avaliar as alterações hematológicas de pacientes com quadro clínico de dengue. Foram estudados 543 prontuários de atendimentos referentes à epidemia pelo vírus tipo 3, ocorrida no ano de 2007, em Campo Grande, Mato Grosso do Sul. Houve predomínio de casos de dengue clássico (90,2\%), com quadro clínico leve sem complicações. As principais alterações hematológicas observadas foram a leucopenia (68,3\%), plaquetopenia (66,5\%), linfocitopenia $(67,2 \%)$ e presença de linfócitos atípicos (67\%). A febre hemorrágica do dengue apresentou plaquetopenia mais prolongada e maior número de linfócitos atípicos, as demais alterações hematológicas apresentaram evolução diária semelhante às encontradas no dengue clássico. As alterações hematológicas observadas no dengue apresentaram-se de acordo com a evolução clínica e gravidade da doença.
\end{abstract}

Palavras-chaves: Dengue. Leucopenia. Trombocitopenia. Linfocitose.

\section{ABSTRACT}

Dengue is a neglected disease with high morbidity and mortality among children and adults that occurs mainly in tropical and subtropical regions. The objective of this study was to evaluate hematological changes in patients with clinical manifestations of dengue. Medical records relating to 543 cases of dengue virus 3 that occurred during the 2007 epidemic in Campo Grande, Mato Grosso do Sul, were studied. Cases of classic dengue predominated (90.2\%), with mild clinical manifestations lacking complications. The main hematological findings were leukopenia (68.3\%), thrombocytopenia (66.5\%), lymphocytopenia (67.2\%) and atypical lymphocytes (67\%). In dengue hemorrhagic fever, thrombocytopenia was more prolonged and the number of atypical lymphocytes was higher, while the other hematological abnormalities presented daily evolution similar to those in classic dengue. The hematological changes observed in dengue present according to the clinical course of the disease and its severity.

Key-words: Dengue. Leukopenia. Thrombocytopenia. Lymphocytosis.

0 dengue é uma enfermidade de importância mundial que acompanha a humanidade há centenas de anos, sendo, no entanto, negligenciada e sem perspectivas de controle em curto prazo $^{11}$. Inúmeras são as epidemias que ainda ocorrem no mundo e no Brasil. Em 2007, o Estado do Mato Grosso do Sul evidenciou sua maior epidemia de dengue, totalizando 69.378 notificações, sendo que na Cidade de Campo Grande, foram registrados 44.695 casos, resultando no maior número de casos por habitante já visto em uma capital brasileira, com um caso de dengue para aproximadamente 16 habitantes $^{48}$. Na epidemia de dengue há interferência na economia de países, pois acomete grande número de pessoas, gera hospitalizações e traz prejuízos não apenas à saúde pública, mas também à

\footnotetext{
1. Curso de Mestrado em Doenças Infecciosas e Parasitárias, Universidade Federal de Mato Grosso do Sul, Campo Grande, MS. 2. Departamento de Tecnologia de Alimentos e Saúde Pública, Universidade Federal de Mato Grosso do Sul, Campo Grande, MS. 3. Departamento de Clínica Médica, Universidade Federal de Mato Grosso do Sul, Campo Grande, MS.

Endereço de correspondência: Dra. Éveny Cristine Luna de Oliveira. Rua Texaco 207, Jockey Club, 79080-090 Campo Grande, MS.

Tel: $55673382-4541$

e-mail: evenycristine@yahoo.com.br

Recebido para publicação em 05/08/2009

Aceito em 09/11/2009
}

cadeia produtiva trabalhista. 0 espectro clínico vai desde um quadro leve, com resolução média em sete dias, até presença de falência hemodinâmica e óbito ${ }^{15}$.

Constantemente observam-se repercussões hematológicas nos pacientes com dengue. São comuns as alterações no hemograma, como hemoconcentração, leucopenia, plaquetopenia e alterações de hemostasia sanguínea com presença frequente de manifestações hemorrágicas. Algumas dessas alterações estão relacionadas com a gravidade da doença e indicam a necessidade de intervenção terapêutica com finalidade de reduzir a mortalidade ${ }^{15}$. 0 objetivo deste trabalho foi analisar as alterações de hemogramas de pacientes com dengue, a fim de identificar as variações encontradas segundo os dias de evolução dessa patologia e o tipo de dengue.

\section{MATERIAL E MÉTODOS}

Foram avaliados 543 prontuários de pacientes atendidos no Hospital-dia Professora Esterina Corsini, unidade referenciada para o atendimento de casos de dengue, Núcleo do Hospital Universitário/Fundação Universidade Federal de Mato Grosso do Sul (NHU/FUFMS), Campo Grande, no período de janeiro a maio de 2007. Os dados referiram-se a 10 dias de acompanhamento clínico. 
Foi definido como caso de dengue, todo indivíduo com doença febril aguda com duração máxima de sete dias, acompanhada de pelo menos dois dos seguintes sintomas: cefaleia, dor retrorbital, mialgia, artralgia, prostração e/ou exantema. Foram considerados os seguintes tipos de dengue: dengue clássico (DC) e febre hemorrágica do dengue (FHD), segundo a classificação da Organização Mundial da Saúde ${ }^{15}$.

Os critérios de exclusão foram os seguintes: caso suspeito com diagnóstico laboratorial negativo para amostras IgM, caso suspeito com diagnóstico de outra entidade clínica e caso suspeito sem confirmação laboratorial, cujas investigações clínica e epidemiológica eram compatíveis com outras patologias.

Foi realizada a estatística descritiva, constando de apresentação tabular e gráfica. Para verificar a associação entre as variáveis do estudo foi utilizado o teste qui-quadrado, ao nível de significância de 5\%. A análise dos dados foi realizada através dos programas Microsoft ${ }^{\circledR}$ Excel 1998, Bioestat e Epi-info 3.4.5. Este estudo (protocolo $\mathrm{n}^{0} 1140$ ) foi aprovado pelo Comitê de Ética e Pesquisa da Universidade Federal de Mato Grosso do Sul, no dia 24 de abril de 2008.

\section{RESULTADOS}

A população analisada foi de 543 pacientes com predomínio de indivíduos do sexo feminino $(57,1 \%)$ e na faixa etária entre 13 a 48 anos, com idade mínima de 10 anos e máxima de 87 anos. A ocorrência de pequeno número de indivíduos com idade inferior a 12 anos deveu-se à existência de um centro específico de referência para pediatria. Do total de pacientes, 90,2\% (IC 95\% 87,7 - 92,7) apresentaram DC e 9,8\% (IC 95\% 7,3 - 12,3) tiveram FHD. Não houve associação entre frequência das diferentes formas clínicas, sexo e faixa etária (Tabela 1).

Não houve caso de FHD de gravidade clínica máxima (grau IV) ou de óbito. Os quadros clínicos considerados mais leves foram mais frequentes no DC (46,9\%). A prova do laço foi realizada na maioria $\left(\mathrm{n}^{\mathrm{0}}=351\right)$ dos pacientes com positividade baixa $(33,3 \%)$.

TABELA 1

Número e porcentagem de pacientes segundo sexo, faixa etária e tipo de dengue (classificação da OMS), Campo Grande - 2007.

\begin{tabular}{|c|c|c|c|c|c|c|c|}
\hline \multirow[b]{2}{*}{ Variáveis } & \multicolumn{2}{|c|}{$\begin{array}{l}\text { Dengue } \\
\text { clássico } \\
\left(\mathrm{n}^{-} 490\right)\end{array}$} & \multicolumn{2}{|c|}{$\begin{array}{c}\text { Febre } \\
\text { hemorrágica } \\
\left(n^{\circ} 53\right)\end{array}$} & \multicolumn{2}{|c|}{$\begin{array}{c}\text { Total } \\
\left(n^{\circ} 543\right)\end{array}$} & \multirow[b]{2}{*}{$\mathrm{p}^{*}$} \\
\hline & $\mathrm{n}^{\underline{0}}$ & $\%$ & $\mathrm{n}^{0}$ & $\%$ & $\mathrm{n}^{0}$ & $\%$ & \\
\hline \multicolumn{8}{|l|}{ Sexo } \\
\hline feminino & 279 & 90,0 & 31 & 10,0 & 310 & 57,1 & 0,944 \\
\hline masculino & 211 & 90,6 & 22 & 9,4 & 233 & 42,9 & \\
\hline \multicolumn{8}{|c|}{ Faixa etária (anos) } \\
\hline$\leq 12$ & 15 & 88,2 & 2 & 11,8 & 17 & 3,1 & 0,121 \\
\hline 13 a 24 & 144 & 94,7 & 8 & 5,3 & 152 & 28,0 & \\
\hline 25 a 36 & 114 & 87,7 & 16 & 12,3 & 130 & 23,9 & \\
\hline 37 a 48 & 102 & 88,7 & 13 & 11,3 & 115 & 21,2 & \\
\hline 49 a 60 & 85 & 92,4 & 7 & 7,6 & 92 & 16,9 & \\
\hline$>60$ & 30 & 81,1 & 7 & 18,9 & 37 & 6,8 & \\
\hline
\end{tabular}

${ }^{*} \mathrm{p} \leq$ 0,05: diferença estatisticamente significativa. Teste qui-quadrado.
Dos 543 prontuários avaliados, 68,5\% apresentaram número de plaquetas inferior a $150.000 / \mathrm{mm}^{3}$. Houve queda do número de plaquetas a partir do $3^{\circ}$ dia no DC e a partir do $1^{\circ}$ e $2^{\circ}$ dias na FHD. A mediana dos valores de plaquetas foram menores na FHD desde o primeiro dia de sintomatologia em relação à DC. Porém, evolução diária semelhante atingindo o menor número no $7^{\circ}$ dia e recuperando-se a partir daí (Figura 1).

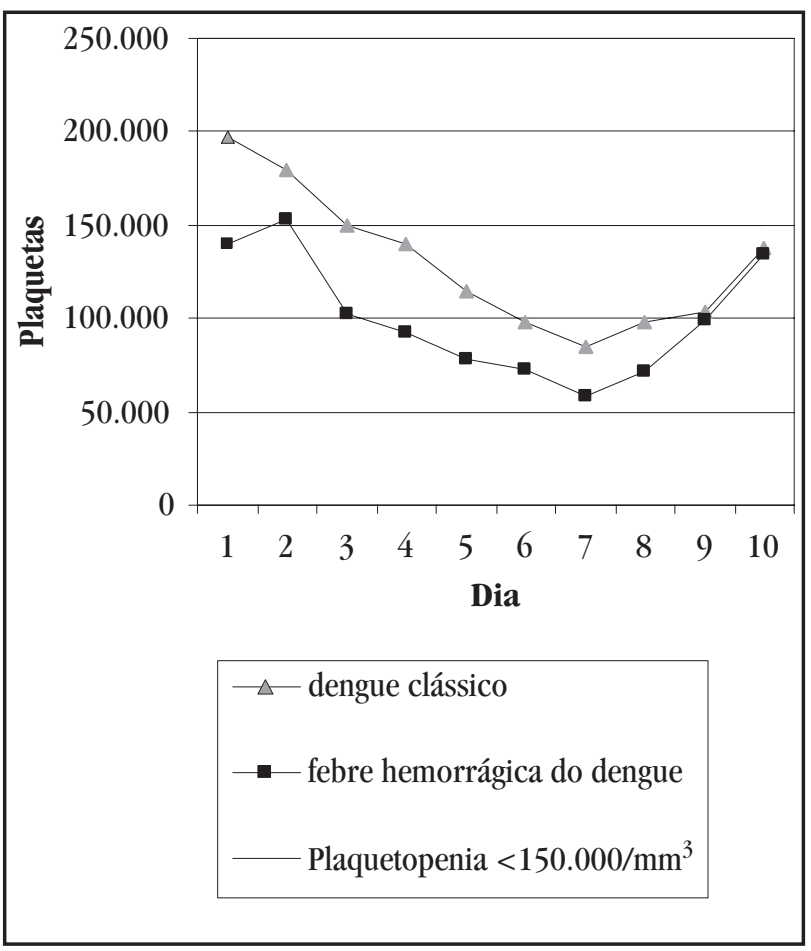

\section{FIGURA 1}

Plaquetas (mediana) segundo o tipo de dengue (classificação da OMS), Campo Grande, 2007.

Do total $\left(\mathrm{n}^{0}=543\right)$ de pacientes, 191 apresentaram hemorragia. No grupo de pacientes no qual houve sangramento, a mediana de plaquetas foi de $95.000 / \mathrm{mm}^{3}$ e no sem sangramento, a mediana foi de $135.000 / \mathrm{mm}^{3}$. O principal tipo de sangramento observado foram as petéquias $(64,4 \%)$, tanto no DC como na FHD.

Considerando somente os 191 pacientes que tiveram hemorragia, dos 45 pacientes com FHD, 21 (46,7\%) apresentaram sangramento em um local, 16 (35,5\%) em dois locais, 8 (17,8\%) em três ou mais locais diferentes. Dos 146 pacientes com DC, 111 (76\%) apresentaram sangramento em um local, 31 (21,2\%) em dois locais e $4(2,7 \%)$ em três locais. Houve diferença estatisticamente significativa $(p<0,001$ - teste qui-quadrado).

Quanto ao número de leucócitos, observou-se que 69,8\% dos pacientes apresentaram número de leucócitos inferior a $4.000 / \mathrm{mm}^{3}$. A leucopenia apresentou-se mais precocemente na FHD, sendo observada já no $2^{\circ}$ dia de sintomatologia e recuperando-se a partir do $8^{\circ}$ dia. No DC, a leucopenia foi evidenciada do $3^{\circ}$ ao $8^{\circ}$ dia com recuperação semelhante à ocorrida na FHD (Figura 2).

Dos 543 pacientes, ocorreu linfopenia em $67,8 \%$, sendo que $363(66,9 \%)$ apresentaram linfócitos atípicos. A porcentagem de linfócitos no sangue periférico variou de 1\% a 35\%. Tanto 


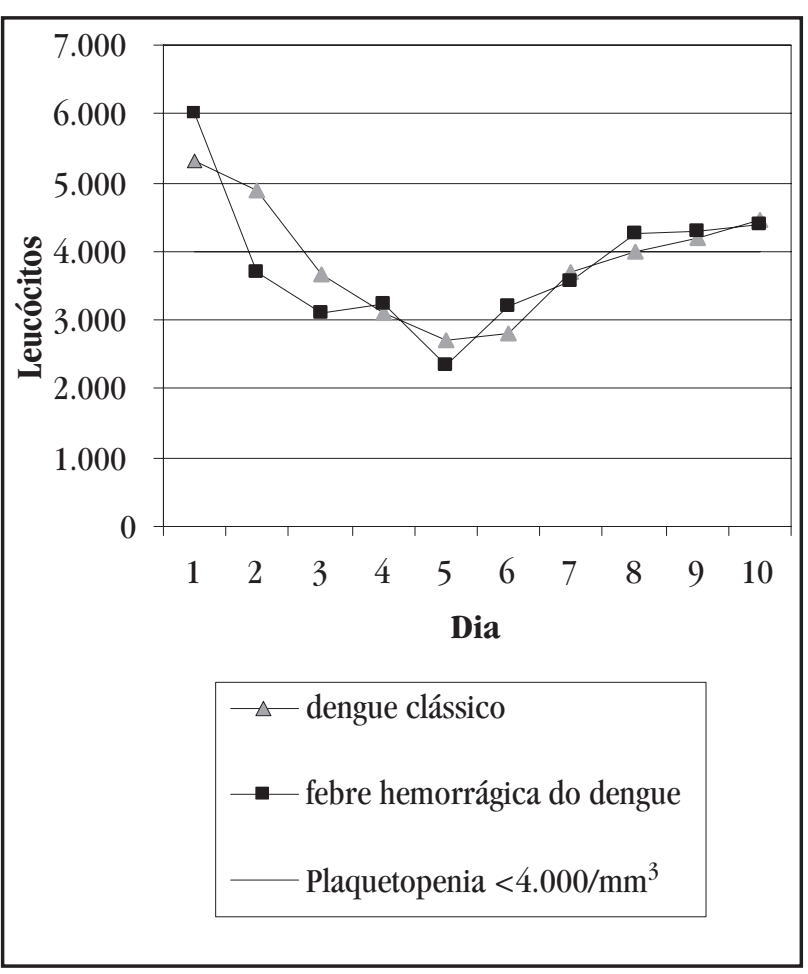

FIGURA 2

Leucócitos (mediana) segundo o tipo de dengue (classificação da OMS), Campo Grande, 2007.

no DC, quanto na FHD, houve normalização do número de linfócitos a partir do sétimo dia (Figura 3); no entanto, o número de linfócitos atípicos aumentou acentuadamente a partir do quinto dia, principalmente na FHD (Figura 4).

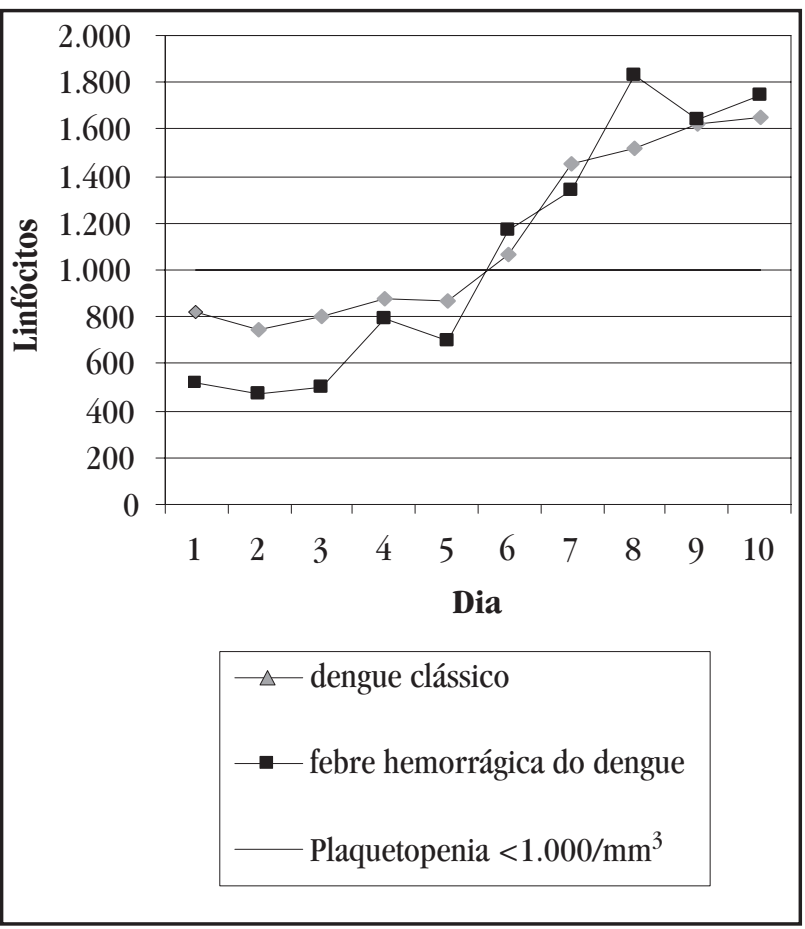

FIGURA 3

Linfócitos (mediana) segundo o tipo de dengue (classificação da OMS), Campo Grande, 2007.

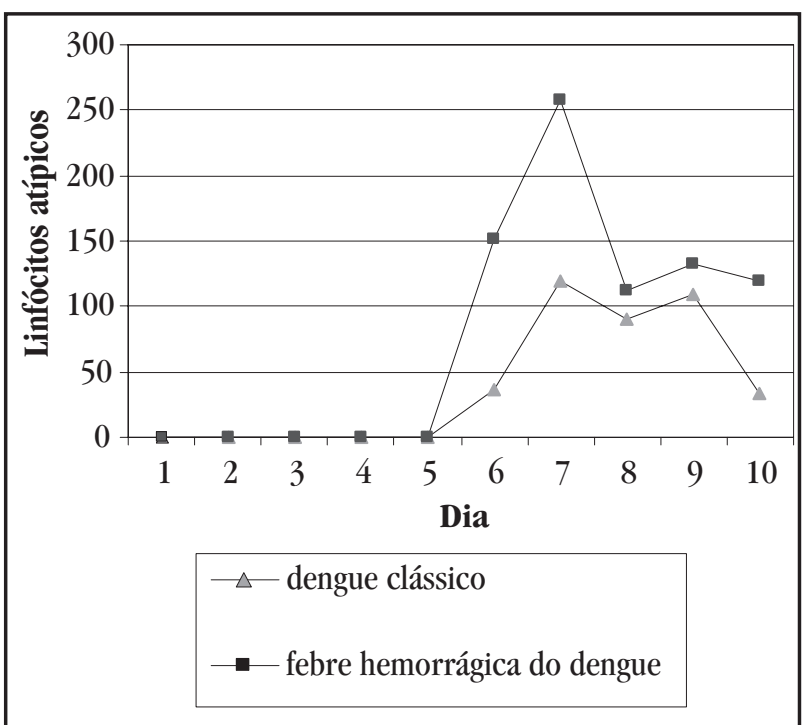

FIGURA 4

Linfócitos atípicos (mediana) segundo o tipo de dengue (classificação da OMS), Campo Grande, 2007.

\section{DISCUSSÃO}

Foi observada alta frequência de leucopenia $(69,8 \%)$ e de trombocitopenia $(68,5 \%)$ neste estudo. Esses resultados foram similares aos de Ayyub e cols ${ }^{1}$, que mostraram a ocorrência de $79,5 \%$ de plaquetopenia e de $48,73 \%$ de leucopenia ${ }^{1}$. Ao contrário, diferem daqueles encontrados em 2008 no Pará, no qual a presença de leucopenia foi de $25,2 \%$, de plaquetopenia foi de $24,3 \%$ e de ambos foi de $13,3 \%^{3}$.

0 grupo com sangramento apresentou número médio de plaquetas superior a $50.000 / \mathrm{mm}^{3}$, nível este raramente associado à clínica de hemorragia espontânea. 0 fato de haver sangramento, mesmo em vigência de número de plaquetas considerado de baixo risco para hemorragia, sugere a presença de outros fatores relacionados à manifestação hemorrágica, tais como: o comprometimento vascular, a disfunção plaquetária e os distúrbios da coagulação sanguínea, como por exemplo, a coagulação intravascular disseminada ${ }^{13}$.

0 grupo FHD apresentou plaquetopenia de maior vulto e maior número de pacientes com mais de um local de sangramento, 0 que pode estar associado a aumento de consumo plaquetário em decorrência de coagulação intravascular disseminada, maior ocorrência de anticorpos antiplaquetários ou de destruição plaquetária por complemento ${ }^{13}$.

Os sangramentos mais frequentemente observados foram os relacionados ao comprometimento da primeira fase da hemostasia, que propicia sangramentos preferencialmente cutâno-mucosos. Resultados similares foram relatados em estudo realizado em 105 pacientes pediátricos com dengue, em Neiva, Colômbia, no qual se verificou a ocorrência de manifestações hemorrágicas de pele e mucosas em $75 \%$ dos pacientes ${ }^{10}$. Não houve diferença quanto ao tipo de sangramento no DC e na FHD, sugerindo que ambos os grupos apresentaram como principal defeito de hemostasia 0 comprometimento de primeira fase. 
A prova do laço é recomendada pela OMS para caracterização do quadro de febre hemorrágica do dengue, porém tem sua utilização limitada pelo desconforto que provoca no paciente. Esse método não foi realizado em todos os pacientes e apresentou positividade baixa. Isso pode indicar que acrescenta pouco ao diagnóstico de febre hemorrágica do dengue, sendo utilizado principalmente como indicador de presença de fragilidade vascular'.

Sabe-se que a ocorrência de leucocitose no dengue pode ser considerada como um fator prognóstico associado ao desenvolvimento de complicações ${ }^{14}$. Em estudo no qual foram avaliadas amostras de sangue de sessenta pacientes com presença de leucopenia durante o dengue, observou-se redução da contagem absoluta de células CD8 e CD4 tanto durante fase aguda da doença quanto em período de convalescença ${ }^{2}$.

Do total de 543 pacientes, houve linfopenia em 67,8\%. Conforme estudo de Liu e cols ${ }^{6}$, a maioria dos pacientes com linfopenia apresenta redução no número de linfócitos T, principalmente CD4, sabendo-se que $80 \%$ dos linfócitos circulantes são linfócitos T e destes, 2/3 são linfócitos $\mathrm{CD}^{6}{ }^{6}$.

Os linfócitos atípicos são definidos como formas intermediárias de ativação dos linfócitos T em decorrência de estímulos antigênicos virais ${ }^{12}$. Observou-se neste trabalho, que 66,9\% dos 543 pacientes apresentaram linfócitos atípicos no sangue periférico, sendo esse achado mais comum na FHD. Estudo realizado em Taiwan, demonstrou linfocitose atípica em 49\% dos pacientes com predomínio desses linfócitos nos indivíduos com FHD, além de inversão da razão CD4/CD87.

Quando se compara o dengue com outras patologias infecciosas virais, de quadro clínico semelhante, constata-se que a presença de no mínimo $10 \%$ de linfócitos atípicos no sangue periférico é bom indicador para diagnóstico de dengue. Esses resultados são consistentes com análises de marcadores celulares que demonstraram predomínio de linfócitos atípicos na febre hemorrágica do dengue através da citometria de fluxo. Esses linfócitos atípicos são, na maioria, linfócitos CD 19 positivos, além disso, são mais frequentes no dia da alta do que na admissão do paciente, relacionando-se com o início da fase de convalescença da doença ${ }^{5}$.

Em conclusão, as principais alterações hematológicas foram: a leucopenia, a plaquetopenia, a linfopenia e a presença de linfócitos atípicos. A febre hemorrágica do dengue apresentou linfopenia e plaquetopenia mais acentuadas e maior número de linfócitos atípicos. As alterações hematológicas da FHD apresentaram evolução diária semelhante às encontradas no dengue clássico, exceto a plaquetopenia, que ocorreu mais precocemente na $\mathrm{FHD}$.

\section{REFERÊNCIAS}

1. Ayyub M, Khazindar AM, Lubbad EH, Barlas S, Alfi AY, Al-Ukayli S. Characteristics of dengue fever in a large public hospital, Jeddah, Saudi Arabia. Journal Ayub Medical College 18:9-13, 2006.

2. Azeredo EL, Zagne S, Alvarenga A, Nogueira RMR, Kubelka CF, Oliveira-Pinto LM. Activated peripheral lymphocytes with increased expression of cell adhesion molecules and cytotoxic markers are associated with dengue fever disease. Memórias do Instituto Oswaldo Cruz 101:437-449, 2006.

3. Barros LPS, Igawa SES, Jocundo AY, Brito Júnior LC. Análise crítica dos achados hematológicos e sorológicos de pacientes com suspeita de dengue. Revista Brasileira de Hematologia e Hemoterapia 30:363-366, 2008.

4. Instituto Brasileiro de Geografia e Estatística. População recenseada e estimada, segundo os municípios - Mato Grosso do Sul - 2007. Censos 2007, Brasília, Dez. 2007. Disponível em:<http://www.ibge.gov.br/home/estatistica/população/ contagem2007/contagem_final/tabela1124.pdf> [acesso em: 11 mai. 2009].

5. Jampangern W, Vongthoung K, Jittmittraphap A, Worapongpaiboon S, Limkittikul K, Chuansumrit A, Tarunotai U, Chongsa-Nguan M. Characterization of atypical lymphocytes and immunophenotypes of lymphocytes in patients with dengue virus infection. Asian Pacific Journal of Allergy and Immunology 25:27-36, 2007.

6. Kipps TJ. Functions of T lymphocytes: T-cell receptors for antigen. In: Beutler E, Litchman MA, Coller BS, Kipps TJ, Seligsohn U (orgs) Williams Hematology. $6^{\text {th }}$ edition, McGraw-Hill, p. 949-963, 2001.

7. Liu CC, Huang KJ, Lin YS, Yeh TM, Liu HS, Lei HY. Transient CD4/CD8 ratio inversion and aberrant immune activation during dengue virus infection. Journal of Medical Virology 68:241-252, 2002.

8. Ministério da Saúde. Casos de Dengue. Brasil, Grandes Regiões e Unidades Federadas, 1997 a 2008. Brasília, 2009. Disponível em: < http://portal.saude. gov.br/portal/arquivos/pdf/tabela_casos_dengue_classico_2008.pdf> [acesso em 10 maio 2009].

9. Phuong CXT, Nhan NT, Wills B, Kneen R, Ha NTT, Mai TTT, Huynh TTT, Lien DTK, Solomon T, Simpson JA, White NJ, Farrar JJ. Evaluation of the World Health Organization standard tourniquet test and a modified tourniquet test in the diagnosis of dengue infection in Vietnam. Tropical Medicine and International Health 7:125-132, 2002.

10. Salgado DM, Rodríguez JA, Garzón M. Caracterización clinica y epidemiologica de dengue hemorragico en Neiva, Colombia 2004. Revista de Salud Pública 9:53-63, 2007.

11. Schatzmayr HG. Viroses emergentes e re-emergentes. Cadernos de Saúde Pública 17:209-213, 2001.

12. Simon MW. The atypical lymphocyte. International Pediatrics 18:20-22, 2003.

13. Srichaikul T, Nimmannitya S. Haematology in dengue and dengue haemorrhagic fever. Best Practice \& Research Clinical Haematology 13:261-276, 2000.

14. Torres EM. Dengue. Estudos Avançados 22:33-52, 2008.

15. World Health Organization. Dengue haemorrhagic fever: diagnosis, treatment, prevention and control. Geneve, WHO, 1997. 